\title{
INEQUALITIES OF THE HERMITE-HADAMARD \\ TYPE FOR QUASI-CONVEX FUNCTIONS VIA THE $(k, s)$-RIEMANN-LIOUVILLE FRACTIONAL INTEGRALS
}

\author{
EZE R. NWAEZE
}

\begin{abstract}
Recently, Hussain et al. in the paper [Some $k$-fractional associates of Hermite-Hadamard's inequality for quasi-convex functions and applications to special means, Fractional Differential Calculus, 7(2) 2017, 301-309] established some new Hermite-Hadamard type inequalities for functions whose absolute values are quasi-convex via the $k$-Riemann-Liouville fractional integral operators. The purpose of this article is to extend and generalize the results, obtained in the aforementioned paper, via the $(k, s)$-fractional integrals.
\end{abstract}

Mathematics subject classification (2010): 26A33, 26D15, 26A51.

Keywords and phrases: Hermite-Hadamard inequality, quasi-convex function, $(k, s)$-Riemann-Liouville fractional integrals, Hölder's inequality, power mean inequality.

\section{REFERENCES}

[1] P. Agarwal, M. Jleli And M. Tomar, Certain Hermite-Hadamard type inequalities via generalized k-fractional integrals, J. inequal. and Appl. 2017 2017:55.

[2] S. S. Dragomir and C. E. M. Pearce, Quasi-convex functions and Hadamard's inequality, Bull. Austral. Math. Soc. 57 (1998), 377-385.

[3] S. S. Dragomir and C. E. M. Pearce, Selected Topics on Hermite-Hadamard Inequalities and Applications, RGMIA Monograph, Victoria University, 2000.

[4] J. HADAMARD, Étude sur les propriétés des fonctions entiéres et en particulier d'une fonction considérée par Riemann, J. Math. Pures Appl. 9 (1893), 171-216.

[5] R. Hussain, A. Ali, A. Latif And G. Gulshan, Some k-fractional associates of HermiteHadamard's inequality for quasi-convex functions and applications to special means, Fractional Differ. Calc. 7 (2) (2017), 301-309.

[6] D. A. Ion, Some estimates on the Hermite-Hadamard inequality through quasi-convex functions, Annals of University of Craiova, Math. Sci. Ser. 34 (2007), 82-87.

[7] M. Jleli, D. O. Regan And B. Samet, On Hermite-Hadamard type inequalities via generalized fractional integrals, Turk. J. Math. 40 (2016), 1221-1230.

[8] A. A. Kilbas, H. M. SRivastava and J. J. Trujillo, Theory and Applications of Fractional Differential Equations, Elsevier Amsterdam 2006.

[9] S. Mubeen and G. M. Habibullah, $k$-fractional integrals and applicatons, Int. J. Contemp. Math. Sciences 7 (2) (2012), 89-94

[10] E. R. Nwaeze AND D. F. M. Torres, Novel results on Hermite-Hadamard kind inequalities for $\eta$-convex functions by means of $(k, r)$-fractional integral operators, arXiv: 1802.05619v1.

[11] M. Z. SARIKAYa, Z. Dahmani, M. E. Kiris And F. Ahmad, $(k, s)$-Riemann-Liouville fractional integral and applications, Hacet. J. Math. Stat. 45 (1) (2016), 77-89.

[12] E. Set, M. Tomar, M. Z. SARikayA, On generalized Grüss type inequalities via k-RiemannLiouville fractional integral, Appl. Math. Comput. 269 (2015), 29-34.

[13] E. SET AND B. ÇELIK, Fractional Hermite-Hadamard type inequalities for Quasi-convex functions, Ordu Univ. J. Sci. Tech. 6 (1) (2016), 137-149. 
[14] M. Tomar, S. Mubeen And J. CHOI, Certain inequalities associated with Hadamard $k$-fractional integral operators, J. Inequal. Appl. 2016 2016:234. 